\title{
Contact force observer for space robots
}

\author{
Francesco Cavenago $^{1}$, Alessandro M. Giordano ${ }^{2}$ and Mauro Massari ${ }^{1}$
}

\begin{abstract}
In this paper, the problem of estimating a contact wrench at the end-effector for a space robot is addressed. To this aim, a generalized force observer based on a base-joints dynamics is first reviewed. Then, a different formulation is proposed, which is based on a centroidal-joints dynamics. The proposed observer features interesting decoupling properties from the base linear velocity that lead to a more practical and better-performing estimation when limitations in real space scenarios are considered. The two observers are compared and the advantage of the proposed one is shown through a simulation example featuring a free-floating robot composed of a 7 degrees-of-freedom (DOF) arm mounted on a 6DOF moving base.
\end{abstract}

\section{INTRODUCTION}

Many on-orbit missions would potentially take advantage of the use of manipulators [1]. However, their exploitation is still very limited due to the high complexity involved in such missions. Especially, dealing with physical contact between the robot and a target object is considered one of the most critical issue.

Many researchers have addressed the problem of guaranteeing a safe interaction between the robot and the target during and after the contact in close proximity operations [2][3][4]. In this situation, the end-effector comes into contact with the target and the accurate knowledge of the force, that is exchanged, can be a valuable information for the implementation of an effective control strategy. For this reason, the space robot can be equipped with a force-torque sensor duly placed at the wrist. However, if the contact does not occur exactly at the foreseen location, the measurement may be inaccurate [5]. Furthermore, this kind of sensor can not be redundant and thus a failure could jeopardize the successful accomplishment of the task.

Therefore, other approaches have been proposed to estimate the contact force without the need of a dedicated sensor at the end-effector. In [5] the use of the disturbance observer is proposed, while in [6] the force is estimated through the target equations of motion. Both methods require quantities that are not measured directly, as the joint accelerations and the linear velocity of robot base for the former, and the target accelerations for the latter. These quantities could be obtained through numerical differentiation, but they would introduce nonnegligible noise in the estimation process.

\footnotetext{
${ }^{1}$ The authors are with the Department of Aerospace Science and Technology, Politecnico di Milano, 20156, Milano, Italy \{francesco.cavenago, mauro.massari\}epolimi.it

${ }^{2}$ The author is with the Technical University of Munich (TUM), Garching, 85748, Germany, and with the DLR Institute of Robotics and Mechatronics, 82234, Weßling, Germany alessandro.giordano@dlr.de
}

In [7] a residual-based observer is proposed for humanoids. This is an adaptation to floating robots of the wellestablished momentum-based observer, presented in [8]. This generalized force observer computes the linear, angular and joint momentum residuals which turn out to be the estimates of the external generalized forces acting on the floating base and the disturbance joint torques due to a contact. Then, the residuals can be used to estimate the external wrench acting on the robot. The main drawback of the method is the need of a fast and accurate reconstruction of the base linear velocity, which is difficult to obtain in real space applications.

In this paper, the residual-based observer [7] is reviewed and formulated for space robots based on a base-joints dynamics. Then, a new observer is derived, which is based on a centroidal-joints dynamics. The most important feature of this observer is the complete decoupling of the angular and joint momentum residuals from the base linear velocity. This decoupling leads to a more practical and better-performing estimation of the external wrench. Indeed, the proposed method requires only the knowledge of the base angular velocity and control moments, and the joint positions, velocities and torques, which can be acquired at high frequency and feature relatively low noise.

The paper is structured as follows: in Sect. II, the notations, assumptions and the main space robot equations are introduced. In Sect. III, the method in [7] is formulated for space robots and the proposed method is presented. In Sect. IV, a method to reconstruct the external wrench at the endeffector is proposed. In Sect. V, a simulation example is proposed to assess the performance of the observers. Finally, in Sect. VI, the main conclusions are drawn and future works are discussed.

\section{Preliminaries}

\section{A. Problem statement and assumptions}

A space robot can be represented as a multibody system composed of $n+1$ rigid bodies connected with $n$ joints (see Fig. 1). In this paper, only revolute joints are considered. An in-orbit proximity operation is taken as reference scenario. The robot is required to perform a capture of another object or manipulation tasks. In this context, accurate knowledge of the contact force at the end-effector is important to guarantee a safe physical interaction. In this study, no disturbances caused by the environment (e.g. gravity gradient, air drag and magnetic forces) are considered, because they are expected to be considerably smaller than the actuation forces. Note that this is a commonly accepted assumption in space robotics. Finally, the presented observers are formulated considering a contact wrench acting on the end-effector. 


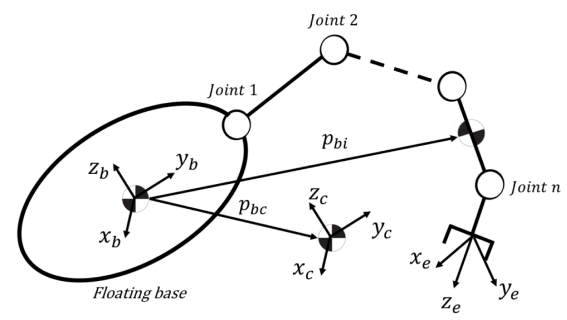

Fig. 1: Floating space robot.

\section{B. Main notations}

Three main reference frames are defined. One, denoted by $\mathcal{B}$, is the body frame located on the center-of-mass (CM) of the spacecraft. The second one, denoted by $\mathcal{C}$, is a frame with rotating axes, parallel to $\mathcal{B}$, placed on the $\mathrm{CM}$ of the whole space robot. The last one, denoted by $\mathcal{E}$, is a frame located on the end-effector.

In order to transform forces and velocities between reference frames, the Adjoint transformation [9] is introduced:

$$
\boldsymbol{A}_{x y}=\left[\begin{array}{cc}
\boldsymbol{R}_{x y} & {\left[\boldsymbol{p}_{x y}\right]^{\wedge} \boldsymbol{R}_{x y}} \\
\mathbf{0} & \boldsymbol{R}_{x y}
\end{array}\right] \in \mathbb{R}^{6 \times 6},
$$

where $\boldsymbol{p}_{x y} \in \mathbb{R}^{3}$ and $\boldsymbol{R}_{x y} \in \mathrm{SO}(3)$ indicate the generic position vector and rotation matrix from frame $\mathcal{X}$ to frame $\mathcal{Y}$, respectively. The operator $[\cdot]^{\wedge}$ stands for the skewsymmetric matrix of the argument. Finally, the identity matrix and zero matrix are denoted by $\boldsymbol{E}$ and $\mathbf{0}$ of suitable dimensions, respectively.

\section{Dynamics model of the impact phase}

The dynamics of the space robot can be expressed as follows:

$$
\begin{aligned}
& \underbrace{\left[\begin{array}{lll}
\boldsymbol{M}_{t} & \boldsymbol{M}_{t r} & \boldsymbol{M}_{t m} \\
\boldsymbol{M}_{t r}^{T} & \boldsymbol{M}_{r} & \boldsymbol{M}_{r m} \\
\boldsymbol{M}_{t m}^{T} & \boldsymbol{M}_{r m}^{T} & \boldsymbol{M}_{m}
\end{array}\right]}_{\boldsymbol{M}(\boldsymbol{q})}\left[\begin{array}{l}
\dot{\boldsymbol{v}}_{b} \\
\dot{\boldsymbol{\omega}}_{b} \\
\ddot{\boldsymbol{q}}
\end{array}\right]+ \\
& +\underbrace{\left[\begin{array}{lll}
\boldsymbol{C}_{t} & \boldsymbol{C}_{t r} & \boldsymbol{C}_{t m} \\
\boldsymbol{C}_{r t} & \boldsymbol{C}_{r} & \boldsymbol{C}_{r m} \\
\boldsymbol{C}_{m t} & \boldsymbol{C}_{m r} & \boldsymbol{C}_{m}
\end{array}\right]}_{\boldsymbol{C}\left(\boldsymbol{v}_{b}, \boldsymbol{\omega}_{b}, \boldsymbol{q}, \dot{\boldsymbol{q}}\right)}\left[\begin{array}{l}
\boldsymbol{v}_{b} \\
\boldsymbol{\omega}_{b} \\
\dot{\boldsymbol{q}}
\end{array}\right]=\left[\begin{array}{c}
\boldsymbol{f}_{b} \\
\boldsymbol{m}_{b} \\
\boldsymbol{\tau}
\end{array}\right]+\left[\begin{array}{c}
\boldsymbol{f}_{\text {ext }, b} \\
\boldsymbol{m}_{\text {ext,b }} \\
\boldsymbol{\tau}_{\text {ext }}
\end{array}\right],
\end{aligned}
$$

with

$$
\left[\begin{array}{c}
\boldsymbol{f}_{e x t, b} \\
\boldsymbol{m}_{e x t, b} \\
\boldsymbol{\tau}_{e x t}
\end{array}\right]=\left[\begin{array}{cc}
\boldsymbol{E} & \mathbf{0} \\
{\left[\boldsymbol{p}_{b e}\right]^{\wedge}} & \boldsymbol{E} \\
\boldsymbol{J}_{v e}^{T} & \boldsymbol{J}_{\omega e}^{T}
\end{array}\right] \mathcal{F}_{e x t},
$$

where $\mathcal{F}_{\text {ext }}=\left[\boldsymbol{f}_{\text {ext }}^{T} \boldsymbol{m}_{\text {ext }}^{T}\right]^{T} \in \mathbb{R}^{6}$ is the contact wrench at the end-effector, expressed in $\mathcal{B} ; \boldsymbol{J}_{v e} \in \mathbb{R}^{3 \times n}, \boldsymbol{J}_{\omega e} \in \mathbb{R}^{3 \times n}$ are the Jacobians mapping $\dot{\boldsymbol{q}}$ into the linear and angular velocity of the end-effector, respectively, considering the base fixed; $\boldsymbol{v}_{b}, \boldsymbol{\omega}_{b} \in \mathbb{R}^{3}$ are the linear and angular velocity of the base expressed in $\mathcal{B} ; \boldsymbol{q}, \dot{\boldsymbol{q}} \in \mathbb{R}^{n}$ are the joint angles and velocities; $\boldsymbol{f}_{b}, \boldsymbol{m}_{b} \in \mathbb{R}^{3}$ are the commanded base force and moment around $\mathcal{B}$, expressed in $\mathcal{B} ; \boldsymbol{\tau} \in \mathbb{R}^{n}$ are the commanded joint torques; the submatrices $\boldsymbol{M}_{t}, \boldsymbol{M}_{t r}, \boldsymbol{M}_{r} \in$ $\mathbb{R}^{3 \times 3}$ compose the inertia matrix of the system regarded as a composite rigid body; the submatrices $\boldsymbol{M}_{t m}, \boldsymbol{M}_{r m} \in \mathbb{R}^{3 \times n}$ are the coupling inertia matrices; $\boldsymbol{M}_{m} \in \mathbb{R}^{n \times n}$ is the inertia matrix of the manipulator; $\boldsymbol{C}\left(\boldsymbol{v}_{b}, \boldsymbol{\omega}_{b}, \boldsymbol{q}, \dot{\boldsymbol{q}}\right) \in \mathbb{R}^{(6+n) \times(6+n)}$ is the Coriolis/centrifugal matrix. The analytical expressions of the inertia matrix can be found in [10].

The total generalized momentum around $\mathcal{B}$ and expressed in $\mathcal{B}$, denoted by $\boldsymbol{h}_{b} \in \mathbb{R}^{6}$, can be written as

$$
\boldsymbol{h}_{b}=\left[\begin{array}{l}
\boldsymbol{h}_{b}^{t} \\
\boldsymbol{h}_{b}^{r}
\end{array}\right]=\left[\begin{array}{ll}
\boldsymbol{M}_{t} & \boldsymbol{M}_{t r} \\
\boldsymbol{M}_{t r}^{T} & \boldsymbol{M}_{r}
\end{array}\right]\left[\begin{array}{l}
\boldsymbol{v}_{b} \\
\boldsymbol{\omega}_{b}
\end{array}\right]+\left[\begin{array}{l}
\boldsymbol{M}_{t m} \\
\boldsymbol{M}_{r m}
\end{array}\right] \dot{\boldsymbol{q}},
$$

with $\boldsymbol{h}_{b}^{t}, \boldsymbol{h}_{b}^{r} \in \mathbb{R}^{3}$ being the translational and rotational momentum, respectively.

\section{FORCE OBSERVER FOR SPACE ROBOTS}

In this section, firstly, the generalized force observer presented in [7] for humanoids is adapted to space robots. This method is based on the momentum-based observer [8] in which a residual vector is defined as the difference between the generalized momentum of the robot and its estimate. Under ideal condition, this residual vector turns out to be a filtered estimation of the external disturbance on the joints. Hereafter, the same idea is followed using the dynamics model (2) to obtain estimates of $\boldsymbol{f}_{e x t, b}, \boldsymbol{m}_{e x t, b}$ and $\boldsymbol{\tau}_{\text {ext }}$. Afterwards, the proposed observer, based on a centroidaljoints dynamics, is derived and discussed. Interesting decoupling properties from the base linear velocity are highlighted, which result in improved performance when real implementation issues are considered.

\section{A. Observer based on a base-joints dynamics}

Considering Eq. (2), the dynamics of the robot can be split into base linear and rotational dynamics, i.e., the first and second rows of the equation, and joint dynamics, i.e., the third row of the equation.

Denoting by $\hat{\boldsymbol{f}}_{\text {ext }, b}, \hat{\boldsymbol{m}}_{\text {ext }, b} \in \mathbb{R}^{3}$ and $\hat{\boldsymbol{\tau}}_{\text {ext }} \in \mathbb{R}^{n}$ the so-called residuals, they are designed as follows:

$$
\begin{aligned}
\hat{\boldsymbol{f}}_{e x t, b}= & \boldsymbol{K}_{f}(\underbrace{\boldsymbol{M}_{t} \boldsymbol{v}_{b}+\boldsymbol{M}_{t r} \boldsymbol{\omega}_{b}+\boldsymbol{M}_{t m} \dot{\boldsymbol{q}}}_{\boldsymbol{h}_{b}^{t}}-\int_{0}^{t}\left(\boldsymbol{f}_{b}+\right. \\
& \left.\left.+\boldsymbol{C}_{t}^{T} \boldsymbol{v}_{b}+\boldsymbol{C}_{r t}^{T} \boldsymbol{\omega}_{b}+\boldsymbol{C}_{m t}^{T} \dot{\boldsymbol{q}}+\hat{\boldsymbol{f}}_{e x t, b}\right) d s\right),
\end{aligned}
$$

$$
\begin{aligned}
& \hat{\boldsymbol{m}}_{e x t, b}= \boldsymbol{K}_{m}(\underbrace{\boldsymbol{M}_{t r}^{T} \boldsymbol{v}_{b}+\boldsymbol{M}_{r} \boldsymbol{\omega}_{b}+\boldsymbol{M}_{r m} \dot{\boldsymbol{q}}}_{\boldsymbol{h}_{b}^{r}}-\int_{0}^{t}\left(\boldsymbol{m}_{b}+\right. \\
&\left.\left.+\boldsymbol{C}_{t r}^{T} \boldsymbol{v}_{b}+\boldsymbol{C}_{r}^{T} \boldsymbol{\omega}_{b}+\boldsymbol{C}_{m r}^{T} \dot{\boldsymbol{q}}+\hat{\boldsymbol{m}}_{e x t, b}\right) d s\right), \\
& \hat{\boldsymbol{\tau}}_{e x t}=\boldsymbol{K}_{\tau}(\underbrace{\boldsymbol{M}_{t m}^{T} \boldsymbol{v}_{b}+\boldsymbol{M}_{r m}^{T} \boldsymbol{\omega}_{b}+\boldsymbol{M}_{m} \dot{\boldsymbol{q}}}_{\boldsymbol{h}_{j}}-\int_{0}^{t}(\boldsymbol{\tau}+ \\
&\left.\left.+\boldsymbol{C}_{t m}^{T} \boldsymbol{v}_{b}+\boldsymbol{C}_{r m}^{T} \boldsymbol{\omega}_{b}+\boldsymbol{C}_{m}^{T} \dot{\boldsymbol{q}}+\hat{\boldsymbol{\tau}}_{e x t}\right) d s\right),
\end{aligned}
$$


where $\boldsymbol{K}_{f}, \boldsymbol{K}_{m} \in \mathbb{R}^{3 \times 3}$ and $\boldsymbol{K}_{\tau} \in \mathbb{R}^{n \times n}$ are positivedefinite diagonal matrices containing the observer gains; $\boldsymbol{h}_{j} \in \mathbb{R}^{n}$ is the joint generalized momentum.

Differentiating Eqs. (5a), (5b), and (5c), and exploiting the dynamics (2) and the property $\dot{\boldsymbol{M}}=\boldsymbol{C}+\boldsymbol{C}^{T}$, the resulting relations between the estimates and the true quantities are

$$
\begin{gathered}
\dot{\hat{\boldsymbol{f}}}_{e x t, b}=\boldsymbol{K}_{f}\left(\boldsymbol{f}_{e x t, b}-\hat{\boldsymbol{f}}_{e x t, b}\right), \\
\dot{\hat{\boldsymbol{m}}}_{e x t, b}=\boldsymbol{K}_{m}\left(\boldsymbol{m}_{e x t, b}-\hat{\boldsymbol{m}}_{e x t, b}\right), \\
\dot{\hat{\boldsymbol{\tau}}}_{e x t}=\boldsymbol{K}_{\tau}\left(\boldsymbol{\tau}_{e x t}-\hat{\boldsymbol{\tau}}_{\text {ext }}\right) .
\end{gathered}
$$

From Eq. (6) it can be noticed that $\hat{\boldsymbol{f}}_{e x t, b}, \hat{\boldsymbol{m}}_{e x t, b}$, and $\hat{\boldsymbol{\tau}}_{\text {ext }}$ are first order estimations of $\boldsymbol{f}_{e x t, b}, \boldsymbol{m}_{e x t, b}$, and $\boldsymbol{\tau}_{e x t}$, respectively.

Increasing the observer gains reduces the time constants of the transient response of the estimates, and thus a faster estimation of $\boldsymbol{f}_{e x t, b}, \boldsymbol{m}_{e x t, b}$, and $\boldsymbol{\tau}_{\text {ext }}$. Ideally, if the observer gains tend to infinity, it would be achieved $\hat{\boldsymbol{f}}_{\text {ext }, b} \approx \boldsymbol{f}_{\text {ext }, b}$, $\hat{\boldsymbol{m}}_{e x t, b} \approx \boldsymbol{m}_{e x t, b}$ and $\hat{\boldsymbol{\tau}}_{e x t} \approx \boldsymbol{\tau}_{\text {ext }}$, respectively. However, in practice, noise and uncertainties induce an upper bound on the values that the observer gains can take. Moreover, the computation of all the residuals, i.e., $\hat{\boldsymbol{f}}_{\text {ext }, b}, \hat{\boldsymbol{m}}_{\text {ext }, b}$, and $\hat{\boldsymbol{\tau}}_{\text {ext }}$ requires the knowledge of the base linear velocity $\boldsymbol{v}_{b}$. A fast and accurate estimation of the linear velocity is particularly difficult in practical applications and the observer scheme (5) would require very low gains resulting in limited response bandwidth. This limitation motivates the derivation of the proposed observer presented hereafter, which turns out to be more practical and better-performing.

\section{B. Observer based on a centroidal-joints dynamics}

In this section, the robot dynamics is transformed using a new set of generalized velocities: the linear velocity of the $\mathrm{CM}$ of the whole system, the angular momentum around $\mathcal{C}$ expressed in $\mathcal{C}$ and the joint velocities. Afterwards, the transformed dynamics is used to formulate the new observer scheme.

The total momentum around $\mathcal{C}$ expressed in $\mathcal{C}$, denoted by $\boldsymbol{h}_{c} \in \mathbb{R}^{6}$, can be found as $\boldsymbol{h}_{c}=\boldsymbol{A}_{c b}^{-T} \boldsymbol{h}_{b}[10]^{1}$, resulting in

$$
\boldsymbol{h}_{c}=\left[\begin{array}{c}
\boldsymbol{h}_{c}^{t} \\
\boldsymbol{h}_{c}^{r}
\end{array}\right]=\left[\begin{array}{ccc}
m \boldsymbol{E} & -m\left[\boldsymbol{p}_{b c}\right]^{\wedge} & m \overline{\boldsymbol{J}}_{v} \\
\mathbf{0} & \boldsymbol{I}_{c} & \boldsymbol{I}_{c} \overline{\boldsymbol{J}}_{\omega}
\end{array}\right]\left[\begin{array}{c}
\boldsymbol{v}_{b} \\
\boldsymbol{\omega}_{b} \\
\dot{\boldsymbol{q}}
\end{array}\right],
$$

where $\boldsymbol{h}_{c}^{t} \in \mathbb{R}^{3}$ is the translational momentum and $\boldsymbol{h}_{c}^{r} \in \mathbb{R}^{3}$ is the rotational momentum around $\mathcal{C}$, both expressed in $\mathcal{C}$, $m \in \mathbb{R}$ and $\boldsymbol{I}_{c} \in \mathbb{R}^{3 \times 3}$ are the mass and the rotational inertia around $\mathcal{C}$ of the whole body, and $\overline{\boldsymbol{J}}_{v}, \overline{\boldsymbol{J}}_{\omega} \in \mathbb{R}^{3 \times n}$ are computed as follows

\footnotetext{
${ }^{1}$ The equations used herein are slightly different from the ones presented in [10]: in [10] the frame $\mathcal{C}$ is nonrotating, whereas here $\mathcal{C}$ is rotating, parallel to $\mathcal{B}$.
}

$$
\begin{gathered}
\overline{\boldsymbol{J}}_{v}=\frac{1}{m} \sum_{i=1}^{n} m_{i} \boldsymbol{R}_{i b}^{T} \boldsymbol{J}_{v i}, \\
\overline{\boldsymbol{J}}_{\omega}=\boldsymbol{I}_{c}^{-1} \sum_{i=1}^{n} \boldsymbol{R}_{i b}^{T} \boldsymbol{I}_{i} \boldsymbol{J}_{\omega i}+m_{i}\left[\boldsymbol{p}_{b i}\right]^{\wedge}\left(\boldsymbol{J}_{v i}-\overline{\boldsymbol{J}}_{v}\right),
\end{gathered}
$$

with $m_{i} \in \mathbb{R}$ and $\boldsymbol{I}_{i} \in \mathbb{R}^{3 \times 3}$ being the mass and rotational inertia of body $i$, computed around its CM, and $\boldsymbol{J}_{v i}, \boldsymbol{J}_{\omega i} \in$ $\mathbb{R}^{3 \times n}$ being the Jacobians mapping $\dot{\boldsymbol{q}}$ in the linear and angular velocity of body $i$, respectively. The Jacobians $\overline{\boldsymbol{J}}_{v}$ and $\overline{\boldsymbol{J}}_{\omega}$ can be also computed based on the inertia model in (2) as:

$$
\begin{gathered}
\overline{\boldsymbol{J}}_{v}=\frac{1}{m} \boldsymbol{M}_{t m} \\
\overline{\boldsymbol{J}}_{\omega}=\left(\boldsymbol{M}_{r}-\frac{1}{m} \boldsymbol{M}_{t r}^{T} \boldsymbol{M}_{t r}\right)^{-1}\left(\boldsymbol{M}_{r m}-\frac{1}{m} \boldsymbol{M}_{t r}^{T} \boldsymbol{M}_{t m}\right) .
\end{gathered}
$$

Introducing the linear velocity of the $\mathrm{CM}$ of the whole system, $\boldsymbol{v}_{c}=\frac{1}{m} \boldsymbol{h}_{c}^{t} \in \mathbb{R}^{3}$, and exploiting Eq. (7), a transformation matrix $\boldsymbol{\Gamma} \in \mathbb{R}^{(6+n) \times(6+n)}$ can be defined as

$$
\left[\begin{array}{c}
\boldsymbol{v}_{c} \\
\boldsymbol{h}_{c}^{r} \\
\dot{\boldsymbol{q}}
\end{array}\right]=\underbrace{\left[\begin{array}{ccc}
\boldsymbol{E} & -\left[\boldsymbol{p}_{b c}\right]^{\wedge} & \overline{\boldsymbol{J}}_{v} \\
\mathbf{0} & \boldsymbol{I}_{c} & \boldsymbol{I}_{c} \overline{\boldsymbol{J}}_{\omega} \\
\mathbf{0} & \mathbf{0} & \boldsymbol{E}
\end{array}\right]}_{\boldsymbol{\Gamma}}\left[\begin{array}{c}
\boldsymbol{v}_{b} \\
\boldsymbol{\omega}_{b} \\
\dot{\boldsymbol{q}}
\end{array}\right] .
$$

Consequently, the generalized forces transform as

$$
\begin{gathered}
{\left[\begin{array}{c}
\boldsymbol{f}_{b} \\
\boldsymbol{m}_{b} \\
\boldsymbol{\tau}
\end{array}\right]=\boldsymbol{\Gamma}^{T}\left[\begin{array}{c}
\boldsymbol{f}_{c} \\
\boldsymbol{a}_{c} \\
\overline{\boldsymbol{\tau}}
\end{array}\right],} \\
{\left[\begin{array}{c}
\boldsymbol{f}_{\text {ext }, b} \\
\boldsymbol{m}_{e x t, b} \\
\boldsymbol{\tau}_{\text {ext }}
\end{array}\right]=\boldsymbol{\Gamma}^{T}\left[\begin{array}{c}
\boldsymbol{f}_{\text {ext }, c} \\
\boldsymbol{a}_{e x t, c} \\
\overline{\boldsymbol{\tau}}_{\text {ext }}
\end{array}\right],}
\end{gathered}
$$

where $\boldsymbol{f}_{c} \in \mathbb{R}^{3}, \boldsymbol{a}_{c} \in \mathbb{R}^{3}$, and $\overline{\boldsymbol{\tau}} \in \mathbb{R}^{n}$ are new control inputs; $\boldsymbol{f}_{\text {ext }, c} \in \mathbb{R}^{3}, \boldsymbol{a}_{\text {ext }, c} \in \mathbb{R}^{3}$, and $\overline{\boldsymbol{\tau}}_{\text {ext }} \in \mathbb{R}^{n}$ are the projections of the external wrench $\mathcal{F}_{\text {ext }}$ into the new variables space.

Then, the dynamics in the new states is derived premultiplying (2) by $\boldsymbol{\Gamma}^{-T}$ and substituting $\boldsymbol{v}_{b}, \boldsymbol{\omega}_{b}, \dot{\boldsymbol{q}}$ and their derivatives isolated from Eq. (12) in (2). The resulting system is

$$
\begin{aligned}
& {\left[\begin{array}{ccc}
m \boldsymbol{E} & \mathbf{0} & \mathbf{0} \\
\mathbf{0} & \boldsymbol{I}_{c}^{-1} & \mathbf{0} \\
\mathbf{0} & \mathbf{0} & \boldsymbol{M}_{m}^{*}
\end{array}\right]\left[\begin{array}{c}
\dot{\boldsymbol{v}}_{c} \\
\dot{\boldsymbol{h}}_{c}^{r} \\
\ddot{\boldsymbol{q}}
\end{array}\right]+} \\
& +\left[\begin{array}{ccc}
\boldsymbol{C}_{c} & \boldsymbol{C}_{c r} & \boldsymbol{C}_{c m} \\
-\boldsymbol{C}_{c r}^{T} & \boldsymbol{C}_{r}^{*} & \boldsymbol{C}_{r m}^{*} \\
-\boldsymbol{C}_{c m}^{T} & -\boldsymbol{C}_{r m}^{* T} & \boldsymbol{C}_{m}^{*}
\end{array}\right]\left[\begin{array}{c}
\boldsymbol{v}_{c} \\
\boldsymbol{h}_{c}^{r} \\
\dot{\boldsymbol{q}}
\end{array}\right]=\left[\begin{array}{c}
\boldsymbol{f}_{c} \\
\boldsymbol{a}_{c} \\
\overline{\boldsymbol{\tau}}
\end{array}\right]+\left[\begin{array}{c}
\boldsymbol{f}_{\text {ext, }, c} \\
\boldsymbol{a}_{\text {ext }, c} \\
\overline{\boldsymbol{\tau}}_{\text {ext }}
\end{array}\right] .
\end{aligned}
$$

Expressing the dynamics in these new variables enables to obtain a system of inertially decoupled dynamic equations. Moreover, it can be demonstrated (see Appendix) that the following relations hold: 


$$
\begin{aligned}
\boldsymbol{C}_{c} \boldsymbol{v}_{c}+\boldsymbol{C}_{c r} \boldsymbol{h}_{c}^{r}+\boldsymbol{C}_{c m} \dot{\boldsymbol{q}}=m\left[\boldsymbol{\omega}_{b}\right]^{\wedge} \boldsymbol{v}_{c}, \\
-\boldsymbol{C}_{c r}^{T} \boldsymbol{v}_{c}+\boldsymbol{C}_{r}^{*} \boldsymbol{h}_{c}^{r}+\boldsymbol{C}_{r m}^{*} \dot{\boldsymbol{q}}=\boldsymbol{I}_{c}^{-1}\left[\boldsymbol{\omega}_{b}\right]^{\wedge} \boldsymbol{h}_{c}^{r}, \\
-\boldsymbol{C}_{c m}^{T} \boldsymbol{v}_{c}-\boldsymbol{C}_{r m}^{* T} \boldsymbol{h}_{c}^{r}+\boldsymbol{C}_{m}^{*} \dot{\boldsymbol{q}}=\left(\dot{\boldsymbol{M}}_{m}^{*}-\frac{1}{2} \boldsymbol{M}_{m / q}^{*}\right) \dot{\boldsymbol{q}}+ \\
+\left(\dot{\overline{\boldsymbol{J}}}_{\omega}^{T}-\overline{\boldsymbol{J}}_{\omega}^{T}\left[\boldsymbol{\omega}_{b}\right]^{\wedge}+\frac{1}{2} \boldsymbol{I}_{c / q}^{-T}-\overline{\boldsymbol{J}}_{\omega / q}^{T}\right) \boldsymbol{h}_{c}^{r},
\end{aligned}
$$

with

$$
\begin{gathered}
\boldsymbol{M}_{m / q}^{*}=\left[\begin{array}{c}
\dot{\boldsymbol{q}}^{T} \frac{\partial \boldsymbol{M}_{m}^{*}}{\partial q_{1}} \\
\vdots \\
\dot{\boldsymbol{q}}^{T} \frac{\partial \boldsymbol{M}_{m}^{*}}{\partial q_{n}}
\end{array}\right] \in \mathbb{R}^{n \times n}, \\
\boldsymbol{I}_{c / q}^{-T}=\left[\begin{array}{c}
\boldsymbol{h}_{c}^{r T} \frac{\partial \boldsymbol{I}_{c}^{-T}}{\partial q_{1}} \\
\vdots \\
\boldsymbol{h}_{c}^{r T} \frac{\partial \boldsymbol{I}_{c}^{-T}}{\partial q_{n}}
\end{array}\right] \in \mathbb{R}^{n \times 3}, \\
\overline{\boldsymbol{J}}_{\omega / q}^{T}=\left[\begin{array}{c}
\dot{\boldsymbol{q}}^{T} \frac{\partial \overline{\boldsymbol{J}}_{\omega}^{T}}{\partial q_{1}} \\
\vdots \\
\dot{\boldsymbol{q}}^{T} \frac{\partial \overline{\boldsymbol{J}}_{\omega}^{T}}{\partial q_{n}}
\end{array}\right] \in \mathbb{R}^{n \times 3} .
\end{gathered}
$$

The dynamic equations can be rewritten as

$$
\begin{aligned}
& m \dot{\boldsymbol{v}}_{c}+m\left[\boldsymbol{\omega}_{b}\right]^{\wedge} \boldsymbol{v}_{c}=\boldsymbol{f}_{c}+\boldsymbol{f}_{e x t, c}, \\
& \dot{\boldsymbol{h}}_{c}^{r}+\left[\boldsymbol{\omega}_{b}\right]^{\wedge} \boldsymbol{h}_{c}^{r}=\boldsymbol{m}_{c}+\boldsymbol{m}_{e x t, c}, \\
& \boldsymbol{M}_{m}^{*} \ddot{\boldsymbol{q}}+\left(\dot{\boldsymbol{M}}_{m}^{*}\right.\left.-\frac{1}{2} \boldsymbol{M}_{m / q}^{*}\right) \dot{\boldsymbol{q}}+\left(\dot{\overline{\boldsymbol{J}}}_{\omega}^{T}-\overline{\boldsymbol{J}}_{\omega}^{T}\left[\boldsymbol{\omega}_{b}\right]^{\wedge}+\right. \\
&\left.+\frac{1}{2} \boldsymbol{I}_{c / q}^{-T}-\overline{\boldsymbol{J}}_{\omega / q}^{T}\right) \boldsymbol{h}_{c}^{r}=\overline{\boldsymbol{\tau}}+\overline{\boldsymbol{\tau}}_{e x t},
\end{aligned}
$$

where it is denoted $\boldsymbol{m}_{c}=\boldsymbol{I}_{c} \boldsymbol{a}_{c} \in \mathbb{R}^{3}$ and $\boldsymbol{m}_{e x t, c}=$ $\boldsymbol{I}_{c} \boldsymbol{a}_{e x t, c} \in \mathbb{R}^{3}$.

First, note that Eqs. (20b) and (20c) do not depend on the base linear velocity anymore. On the other hand, Eq. (20a) depends on the base linear velocity trough $\boldsymbol{v}_{c}$. Second, note that $\boldsymbol{M}_{m}^{*}, \overline{\boldsymbol{J}}_{\omega}, \boldsymbol{M}_{m / q}^{*}, \boldsymbol{I}_{c / q}$, and $\overline{\boldsymbol{J}}_{\omega / q}$ depend only on $\boldsymbol{q}$, and $\dot{\overline{\boldsymbol{J}}}_{\omega}$ depends on $\boldsymbol{q}$ and $\dot{\boldsymbol{q}}$.

Starting from (20), the residuals $\hat{\boldsymbol{f}}_{\text {ext,c }}, \hat{\boldsymbol{m}}_{\text {ext }, c} \in \mathbb{R}^{3}$ and $\hat{\bar{\tau}}_{e x t} \in \mathbb{R}^{n}$, are designed as

$$
\begin{aligned}
\hat{\boldsymbol{f}}_{e x t, c}= & \boldsymbol{K}_{f}(\underbrace{m \boldsymbol{v}_{c}}_{\boldsymbol{h}_{c}^{t}}-\int_{0}^{t}\left(\boldsymbol{f}_{c}-m\left[\boldsymbol{\omega}_{b}\right]^{\wedge} \boldsymbol{v}_{c}+\hat{\boldsymbol{f}}_{e x t, c}\right) d s),(21 a) \\
\hat{\boldsymbol{m}}_{e x t, c}= & \boldsymbol{K}_{m}\left(\boldsymbol{h}_{c}^{r}-\int_{0}^{t}\left(\boldsymbol{m}_{c}-\left[\boldsymbol{\omega}_{b}\right]^{\wedge} \boldsymbol{h}_{c}^{r}+\hat{\boldsymbol{m}}_{e x t, c}\right) d s\right),(21 b \\
\hat{\overline{\boldsymbol{\tau}}}_{e x t}= & \boldsymbol{K}_{\tau}(\overbrace{\boldsymbol{M}_{m}^{*} \dot{\boldsymbol{q}}}^{\boldsymbol{h}_{j}^{*}}-\int_{0}^{t}\left(\overline{\boldsymbol{\tau}}+\frac{1}{2} \boldsymbol{M}_{m / q}^{*} \dot{\boldsymbol{q}}-\left(\dot{\overline{\boldsymbol{J}}}_{\omega}^{T}+\right.\right. \\
& \left.\left.\left.-\overline{\boldsymbol{J}}_{\omega}^{T}\left[\boldsymbol{\omega}_{b}\right]^{\wedge}+\frac{1}{2} \boldsymbol{I}_{c / q}^{-T}-\overline{\boldsymbol{J}}_{\omega / q}^{T}\right) \boldsymbol{h}_{c}^{r}+\hat{\overline{\boldsymbol{\tau}}}_{e x t}\right) d s\right), \quad
\end{aligned}
$$

where $\boldsymbol{h}_{j}^{*} \in \mathbb{R}^{n}$ is the new joint generalized momentum in the centroidal-joints dynamics. Differentiating Eqs. (21a), (21b), and (21c), and using the dynamics (20), the resulting relations between the estimates and the true quantities are

$$
\begin{gathered}
\dot{\hat{\boldsymbol{f}}}_{e x t, c}=\boldsymbol{K}_{f}\left(\boldsymbol{f}_{e x t, c}-\hat{\boldsymbol{f}}_{e x t, c}\right), \\
\dot{\hat{\boldsymbol{m}}}_{e x t, c}=\boldsymbol{K}_{m}\left(\boldsymbol{m}_{e x t, c}-\hat{\boldsymbol{m}}_{e x t, c}\right),
\end{gathered}
$$

$$
\dot{\bar{\tau}}_{e x t}=\boldsymbol{K}_{\tau}\left(\overline{\boldsymbol{\tau}}_{e x t}-\hat{\bar{\tau}}_{e x t}\right),
$$

meaning that $\hat{\boldsymbol{f}}_{\text {ext }, c}, \hat{\boldsymbol{m}}_{e x t, c}$, and $\hat{\overline{\boldsymbol{\tau}}}_{\text {ext }}$ are first order estimations of $\boldsymbol{f}_{\text {ext,c }}, \boldsymbol{m}_{e x t, c}$, and $\overline{\boldsymbol{\tau}}_{\text {ext }}$, respectively. The observations about the gains tuning made in the previous section are also valid for the proposed observer.

Note that the residuals $\hat{\boldsymbol{m}}_{e x t, c}$ and $\hat{\overline{\boldsymbol{\tau}}}_{\text {ext }}$ in (21b) and (21c) are computed without using the linear velocity. Indeed, $\boldsymbol{v}_{b}$ does not appear in (21b) and (21c), and the matrices therein are only function of $\boldsymbol{q}$ and $\dot{\boldsymbol{q}}$, as remarked previously. This property has an important implication from the practical point of view. Considering the on-orbit scenarios, the linear velocity is not directly measured and its accurate estimation or reconstruction is difficult. Thanks to the decoupled structure of (20b) and (20c), the residuals $\hat{\boldsymbol{m}}_{e x t, c}$ and $\hat{\overline{\boldsymbol{\tau}}}_{\text {ext }}$ can be computed just relying on gyroscopes, encoders and torque sensors, which typically feature good acquisition frequency and relatively low noise. The use of star trackers, limited in frequency, and the discrete derivative of lidar or camera measurements, limited in frequency as well and featuring relatively high noise, is totally avoided. Then, the estimates $\hat{\boldsymbol{m}}_{e x t, c}$ and $\hat{\overline{\boldsymbol{\tau}}}_{\text {ext }}$ can be exploited to reconstruct the external wrench $\mathcal{F}_{\text {ext }}$, as it will be shown in the Sect. IV.

\section{EXTERNAL WRENCH RECONSTRUCTION}

Assuming a contact at the end-effector, the relation between the contact generalized forces $\boldsymbol{f}_{\text {ext }, b}, \boldsymbol{m}_{\text {ext }, b}$, and $\boldsymbol{\tau}_{\text {ext }}$ in (2) and the external wrench $\mathcal{F}_{\text {ext }}$ has been reported in Eq. (3). Using the inverse of Eq. (14) and recalling that $\boldsymbol{m}_{e x t, c}=\boldsymbol{I}_{c} \boldsymbol{a}_{e x t, c}$, the relation between $\boldsymbol{f}_{e x t, c}, \boldsymbol{m}_{e x t, c}$, and $\overline{\boldsymbol{\tau}}_{\text {ext }}$ and $\mathcal{F}_{\text {ext }}$ is given by:

$$
\left[\begin{array}{c}
\boldsymbol{f}_{\text {ext }, c} \\
\boldsymbol{m}_{\text {ext }, c} \\
\overline{\boldsymbol{\tau}}_{\text {ext }}
\end{array}\right]=\underbrace{\left[\begin{array}{cc}
\boldsymbol{E} & \mathbf{0} \\
{\left[\boldsymbol{p}_{c e}\right]^{\wedge}} & \boldsymbol{E} \\
\boldsymbol{J}_{c, v e}^{T} & \boldsymbol{J}_{c, w e}^{T}
\end{array}\right]}_{\boldsymbol{J}_{v h c}^{T}} \mathcal{F}_{e x t},
$$

where

$$
\begin{gathered}
\boldsymbol{J}_{c, v e}=-\overline{\boldsymbol{J}}_{v}+\left[\boldsymbol{p}_{c e}\right]^{\wedge} \overline{\boldsymbol{J}}_{\omega}+\boldsymbol{J}_{v e} \in \mathbb{R}^{3 \times n}, \\
\boldsymbol{J}_{c, \omega e}=-\overline{\boldsymbol{J}}_{\omega}+\boldsymbol{J}_{\omega e} \in \mathbb{R}^{3 \times n}
\end{gathered}
$$

Then, an estimate $\hat{\mathcal{F}}_{\text {ext }}$ of the external wrench at the endeffector can be computed as 
TABLE I: Kinematics and dynamics parameters.

$$
\hat{\mathcal{F}}_{\text {ext }}=\left(\left[\begin{array}{cc}
\boldsymbol{E} & \mathbf{0} \\
{\left[\boldsymbol{p}_{c e}\right]^{\wedge}} & \boldsymbol{E} \\
\boldsymbol{J}_{c, v e}^{T} & \boldsymbol{J}_{c, \omega e}^{T}
\end{array}\right]\right)^{\#}\left[\begin{array}{c}
\hat{\boldsymbol{f}}_{\text {ext }, c} \\
\hat{\boldsymbol{m}}_{\text {ext }, c} \\
\hat{\overline{\boldsymbol{\tau}}}_{\text {ext }}
\end{array}\right],
$$

where the operator $(\cdot)^{\#}$ stands for the Moore-Penrose inverse of the argument.

Considering $\boldsymbol{J}_{v h c}$ has maximum rank, one has the freedom to eliminate redundant rows. Especially, the estimate of the external wrench can be obtained using only $\hat{\boldsymbol{m}}_{e x t, c}$ and $\hat{\overline{\boldsymbol{\tau}}}_{e x t}$, and thus getting rid of the base linear velocity measurement. Selecting the last two rows of Eq. (23) and taking the pseudo inverse, the following relation can be written

$$
\hat{\mathcal{F}}_{\text {ext }}=\left(\boldsymbol{J}_{h c}^{T}\right)^{\#}\left[\begin{array}{c}
\hat{\boldsymbol{m}}_{\text {ext }, c} \\
\hat{\overline{\boldsymbol{\tau}}}_{\text {ext }}
\end{array}\right],
$$

with

$$
\boldsymbol{J}_{h c}=\left[\begin{array}{cc}
-\left[\boldsymbol{p}_{c e}\right]^{\wedge} & \boldsymbol{J}_{c, v e} \\
\boldsymbol{E} & \boldsymbol{J}_{c, w e}
\end{array}\right] \in \mathbb{R}^{6 \times(n+3)} .
$$

Clearly, the rank of $\boldsymbol{J}_{h c}$ should be maximum to reconstruct $\hat{\mathcal{F}}_{\text {ext }}$ correctly. Notice that by using Eq. (26), it is possible to estimate the external wrench acting on the end-effector without using any unpractical measurements, such as the base linear velocity or the joint accelerations.

As last remark, note that the proposed observer can be used to detect and reconstruct contacts along generic points of the robot. Indeed, it is sufficient to substitute in Eq. (24) the Jacobians computed for the end-effector, i.e., $\boldsymbol{J}_{v e}$ and $\boldsymbol{J}_{\omega e}$, with the ones computed for the generic point. Afterwards, the resulting relations between the external wrench and the residuals, having the same form of (25) and (26), can be used for the reconstruction.

\section{Simulation example}

In this section, the two observers are compared through a simulation example and the superior performance of the proposed method based on the centroidal-joints dynamics is shown.

\section{A. Simulation scenario}

A 7DOF manipulator mounted on a 6DOF floating base is considered. The kinematics and dynamics parameters are reported in Tab. I.

The system is free-floating, namely the base actuators are turned off, i.e., $\boldsymbol{f}_{b}=\mathbf{0}$ and $\boldsymbol{m}_{b}=\mathbf{0}$, and the manipulator is controlled to keep the initial configuration, i.e., $\boldsymbol{q}_{0}=$ $\left[\begin{array}{lllllll}0 & 40 & 0 & 110 & 0 & 45 & 0\end{array}\right]$ deg. A simple PD controller is implemented for each joint. Note that the performance of both observers does not depend on the control strategy adopted.

A constant external wrench is applied at the end-effector between $t=1 \mathrm{~s}$ and $t=2.1 \mathrm{~s}$. The wrench consists of only a linear force, with value $\boldsymbol{f}_{\text {ext }}=\left[\begin{array}{lll}-10 & 5 & 8\end{array}\right]^{T} \mathrm{~N}$, and no torque.

\begin{tabular}{cccccc}
\hline & $l[\mathrm{~m}]$ & $m[\mathrm{~kg}]$ & $I_{x}\left[\mathrm{kgm}^{2}\right]$ & $I_{y}\left[\mathrm{kgm}^{2}\right]$ & $I_{z}\left[\mathrm{kgm}^{2}\right]$ \\
\cline { 2 - 6 } Base & - & 150 & 15.0 & 21.8 & 18.88 \\
Link 1 & 0.17 & 5 & 0.03 & 0.03 & 0.03 \\
Link 2 & 1.3 & 15 & 1.65 & 1.65 & 0.64 \\
Link 3 & 0.17 & 8 & 0.15 & 0.15 & 0.03 \\
Link 4 & 1.3 & 12 & 0.25 & 0.25 & 0.03 \\
Link 5 & 0.17 & 10 & 0.26 & 0.26 & 0.03 \\
Link 6 & 0.1 & 5 & 0.02 & 0.02 & 0.02 \\
Link 7 & 0 & 4.7 & 0.01 & 0.01 & 0.01 \\
\hline
\end{tabular}

\section{B. Measurement model}

The angular velocity can be directly measured using a gyro and the joint angles are measured by the encoders. On the other hand, the linear velocity, required only by the observer (5), and the joint velocities, required by both observers, are not directly measured but need to be reconstructed, either by using a discrete derivative or by fusion. Herein the problem of the reconstruction is not addressed and a simplified noise performance model has been used for the sake of comparing the performance of the two observer schemes with similar noise conditions. A white Gaussian noise with zero mean is considered. For the angular velocity a bias is also introduced leading to the following model [11]:

$$
\begin{aligned}
\boldsymbol{\omega}_{b}^{m} & =\boldsymbol{\omega}_{b}+\boldsymbol{b}_{\omega}+\boldsymbol{\eta}_{\omega} \\
\dot{\boldsymbol{b}}_{\omega} & =\boldsymbol{\eta}_{b \omega},
\end{aligned}
$$

where $\boldsymbol{\omega}_{b}^{m} \in \mathbb{R}^{3}$ is the measured angular velocity of the base; the term $\boldsymbol{b}_{\omega} \in \mathbb{R}^{3}$ is the bias, considered to be a "Brownian" motion process; the terms $\boldsymbol{\eta}_{\omega}, \boldsymbol{\eta}_{b \omega} \in \mathbb{R}^{3}$ are white Gaussian noise with zero mean. The standard deviations of the considered noise models are $\sigma_{v_{b}}=1 \mathrm{~mm} / \mathrm{s}$, $\sigma_{q}=5 \cdot 10^{-5} \mathrm{rad}, \sigma_{\dot{q}}=10^{-4} \mathrm{rad} / \mathrm{s}, \sigma_{\tau}=0.1 \mathrm{Nm}$, $\sigma_{b \omega}=3.162 \cdot 10^{-4} \mu \mathrm{rad} / \mathrm{s}^{3 / 2}$, and $\sigma_{\omega}=0.316 \mu \mathrm{rad} / \mathrm{s}^{1 / 2}$. The initial bias for each axis of the gyro model is $b_{\omega, 0}=$ $0.0017 \mathrm{rad} / \mathrm{hr}$.

\section{Observers comparison}

Figs. 2 and 3 show a comparison between the reviewed observer [7] and the proposed one. Fig. 2 compares the two observers when all the three residuals are used to reconstruct $f_{\text {ext }}$. In this case, for the observer based on a base-joints dynamics, i.e., given by (5), a contact force estimate $\hat{f}_{\text {ext }}$ is computed by taking the pseudo inverse of the relation (3) and using the residuals $\hat{\boldsymbol{f}}_{\text {ext }, b}, \hat{\boldsymbol{m}}_{\text {ext }, b}$, and $\hat{\boldsymbol{\tau}}_{e x t}$, whereas for the observer (21) it is given by using the relation (25). On the other hand, Fig. 3 compares the two observers when only the angular and joint momentum residuals are used to reconstruct $f_{\text {ext }}$. For the observer (5), the contact force estimate $\hat{f}_{\text {ext }}$ is computed removing the first row of (3), i.e., without $\hat{\boldsymbol{f}}_{\text {ext }, b}$, and by taking the pseudo inverse of the resulting relation, while for the observer (21) it is given by using the relation (26), i.e., without $\hat{f}_{e x t, c}$. In both observers, all the gains are set equal to $20 \mathrm{~s}^{-1}$.

Fig. 2 shows that the estimation process is significantly affected by noise in the measurements and the observers provide similar bad performance. This is caused by the 


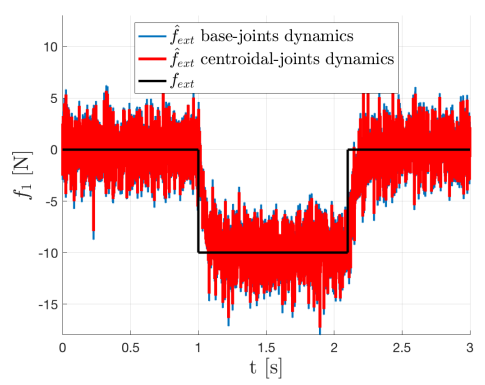

(a) $x$ component.

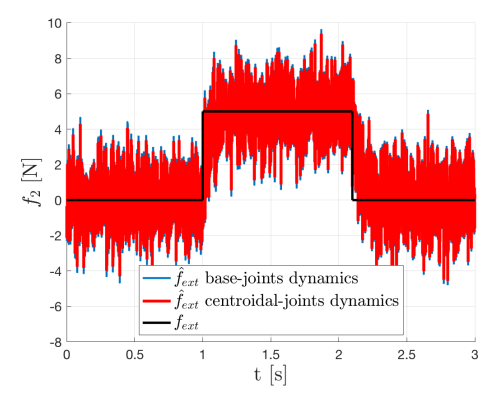

(b) $y$ component.

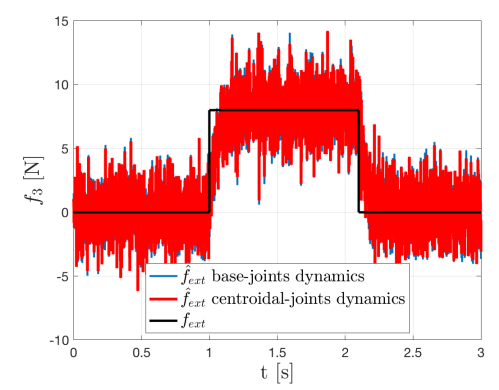

(c) $z$ component.

Fig. 2: Comparison between the reviewed observer [7] and the proposed one, including $\hat{f}_{e x t, b}$ and $\hat{f}_{e x t, c}$ in the estimation process.

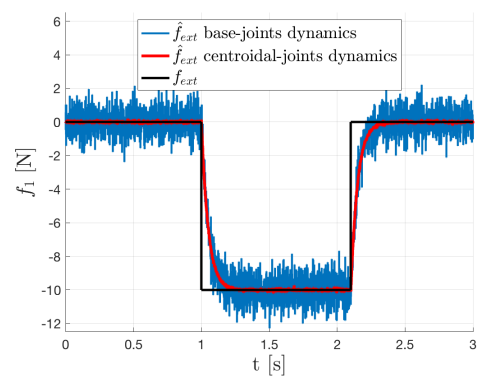

(a) $x$ component.

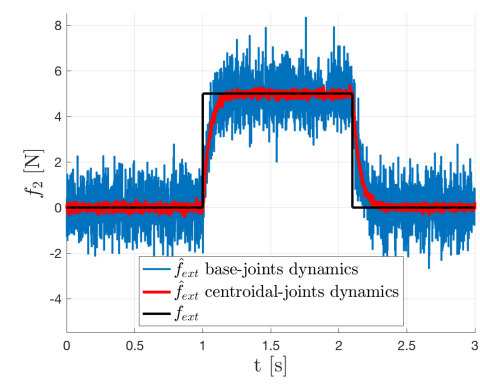

(b) $y$ component.

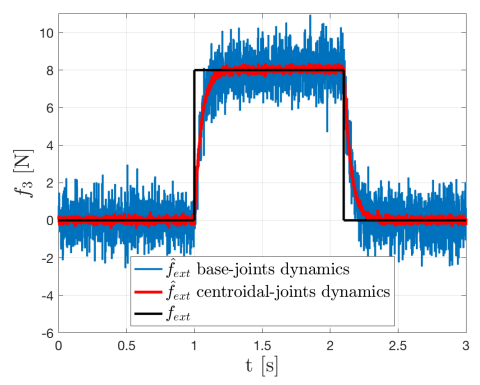

(c) $z$ component.

Fig. 3: Comparison between the reviewed observer [7] and the proposed one, without using $\hat{\boldsymbol{f}}_{\text {ext }, b}$ and $\hat{\boldsymbol{f}}_{\text {ext }, c}$ in the estimation process.

residuals $\hat{\boldsymbol{f}}_{\text {ext }, b}$ and $\hat{\boldsymbol{f}}_{\text {ext }, c}$, which are the residuals mostly affected by noise in the linear velocity. Indeed, in their computation, the linear velocity is multiplied by the total mass of the system and consequently its noise is amplified. On the other hand, the simulation shown in Fig. 3, in which $\hat{f}_{e x t, b}$ and $\hat{f}_{e x t, c}$ are not used in the estimation process, highlights the advantage of using the proposed observer based on the centroidal-joints dynamics. Indeed, it provides a definitely more accurate estimate of the contact force. It is worth stressing that the superior performance of the proposed method derives from the complete decoupling of $\hat{\boldsymbol{m}}_{\text {ext }, c}$ and $\hat{\bar{\tau}}_{\text {ext }}$ from the linear velocity $\boldsymbol{v}_{b}$, which is difficult to reconstruct and, if available, can be particularly noisy and uncertain. The difference in the performance can be even more pronounced if the noise increases. The performance of the observer based on the base-joints dynamics would deteriorate significantly. As a matter of fact, in Fig. 4 the performance of the two observers considering an increased noise level of $3 \mathrm{~mm} / \mathrm{s}$ on $\boldsymbol{v}_{b}$ is shown. To decrease the noise in the estimation process, the observer gains should be strongly reduced, leading to very low response bandwidth. Conversely, the proposed observer is not affected by the increase of noise in the linear velocity, preserving good performance. The observer gains could be even increased, leading to a larger response bandwidth.

\section{Reconstruction of the contact force on a generic point}

In this section, it is shown that the proposed observer based on a centroidal-joints dynamics can be used also to detect and reconstruct a contact force acting on a generic point along the robot. Note that it is assumed to know where the contact occurs. This is a strong assumption since it is not trivial to identify exactly the contact point. Methods are available in the literature to tackle the isolation of the contact point for fixed-base robots and humanoids [7][8]. This problem will be investigated in future works.

Similarly to the previous case, a constant external wrench is applied between $t=1 \mathrm{~s}$ and $t=2.1 \mathrm{~s}$ and it consists of the linear force $\boldsymbol{f}_{\text {ext }}$ and no torque. In this case, the external wrench acts on the $\mathrm{CM}$ of the link 5 .

Known the contact point, the relation (24) should be modified substituting the Jacobians computed for the end-effector, i.e., $\boldsymbol{J}_{v e}$ and $\boldsymbol{J}_{\omega e}$, with the ones computed for the CM of the link 5. Afterwards, the resulting relation, having the form of (26), is used to estimate the contact force $f_{\text {ext }}$. Fig. 5 shows that the proposed observer provides good performance even in the case of a contact on a generic point along the robot.

\section{CONCLUSIONS AND FUTURE WORKS}

The problem of estimating a contact wrench at the endeffector for a space robot was addressed. Two observers were presented. The first one was formulated readapting the 


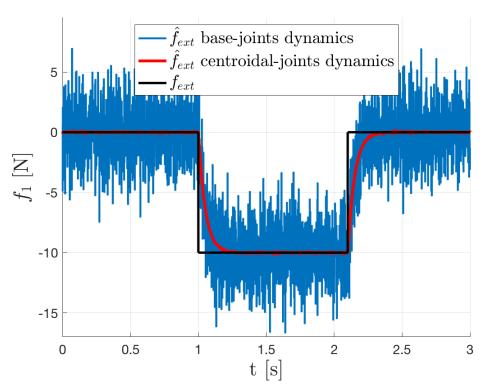

(a) $x$ component.

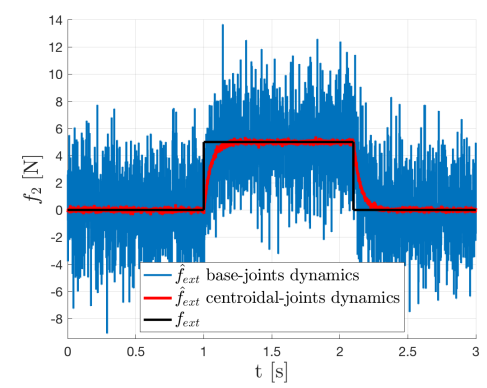

(b) $y$ component.

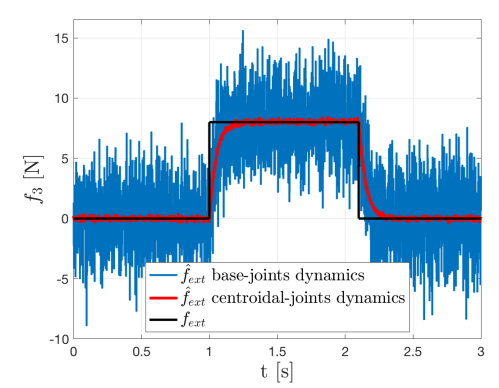

(c) $z$ component.

Fig. 4: Comparison between the reviewed observer [7] and the proposed one, increasing the noise on the linear velocity and without using $\hat{\boldsymbol{f}}_{\text {ext }, b}$ and $\hat{\boldsymbol{f}}_{\text {ext }, c}$ in the estimation process.

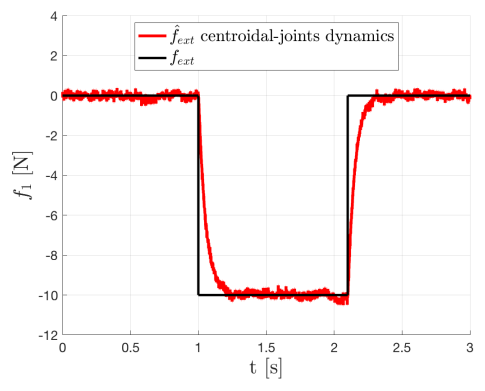

(a) $x$ component.

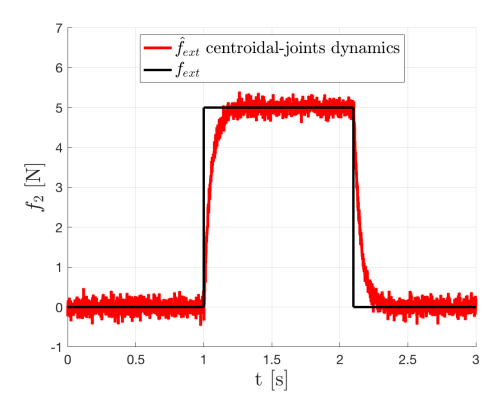

(b) $y$ component.

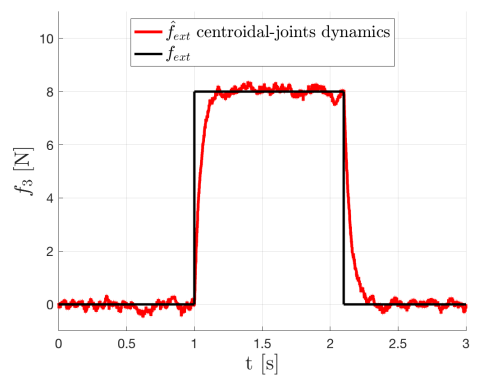

(c) $z$ component.

Fig. 5: Reconstruction of an external wrench acting on the CM of link 5 by the observer based on a centroidal-joints dynamics.

work in [7] to space robots; the second one was derived introducing a new set of generalized velocities, including the linear velocity of the $\mathrm{CM}$ of the whole system, the rotational momentum around $\mathcal{C}$ and the joint velocities. Based on the new generalized velocities, an observer was derived, which does not use any measurement of the linear velocity and can be implemented with realistic sensor measurements. The improved performance of the proposed observer was validated in simulation.

Future works may validate the proposed method on hardware, considering both free-floating and actuated base robots. Furthermore, the problem of the isolation of the contact point along the robot structure may be addressed.

\section{APPENDIX}

In this appendix, the mathematical derivation of the relations (16) is reported.

Deriving the relations (16a) and (16b) is straightforward. Indeed, the differentiation of the momenta $\boldsymbol{h}_{c}^{t}$ and $\boldsymbol{h}_{c}^{r}$, which are expressed in a frame rotating with an angular velocity $\boldsymbol{\omega}_{b}$, results in

$$
\begin{aligned}
\dot{\boldsymbol{h}}_{c}^{t}+\left[\boldsymbol{\omega}_{b}\right]^{\wedge} \boldsymbol{h}_{c}^{t} & =\boldsymbol{f}_{c, t o t} \\
\dot{\boldsymbol{h}}_{c}^{r}+\left[\boldsymbol{\omega}_{b}\right]^{\wedge} \boldsymbol{h}_{c}^{r} & =\boldsymbol{m}_{c, t o t}
\end{aligned}
$$

where $\boldsymbol{f}_{c, t o t} \in \mathbb{R}^{3}, \boldsymbol{m}_{c, t o t} \in \mathbb{R}^{3}$ are the total centroidal force and moment acting on the system. Recalling that $\boldsymbol{h}_{c}^{t}=m \boldsymbol{v}_{c}$ and comparing Eq. (29) with the first row of Eq. (15), the first relation (16a) is obtained. Similarly, comparing Eq. (30) with the second row of Eq. (15), the second relation (16b) is obtained.

For what concerns the third relation (16c), a quasiLagrangian approach [12] is used to rederive the joint equation in (15). Then, the relation (16c) is obtained by comparison of the quasi-Lagrangian joint formulation with the third row of Eq. (15). In the quasi-Lagrangian formulation, the generalized velocities $\boldsymbol{v}_{c}$ and $\boldsymbol{h}_{c}^{r}$ are called quasivelocities since they are not integrable. In order to derive the dynamic equations, some integrable generalized coordinates $\boldsymbol{y} \in \mathbb{R}^{6+n}$ are defined, whose derivatives are related to the quasi-velocities as follows:

$$
\left[\begin{array}{l}
\boldsymbol{v}_{c} \\
\boldsymbol{h}_{c}^{r} \\
\dot{\boldsymbol{q}}
\end{array}\right]=\left[\begin{array}{ccc}
\boldsymbol{E} & -\left[\boldsymbol{r}_{c}\right]^{\wedge} \boldsymbol{J}_{\phi}^{-1} & \mathbf{0} \\
\mathbf{0} & \boldsymbol{I}_{c} \boldsymbol{J}_{\phi}^{-1} & \boldsymbol{I}_{c} \overline{\boldsymbol{J}}_{\omega} \\
\mathbf{0} & \mathbf{0} & \boldsymbol{E}
\end{array}\right]\left[\begin{array}{c}
\dot{\boldsymbol{r}}_{c} \\
\dot{\boldsymbol{\phi}} \\
\dot{\boldsymbol{q}}
\end{array}\right]=\boldsymbol{\Xi}^{T} \dot{\boldsymbol{y}}
$$

where $\boldsymbol{r}_{c} \in \mathbb{R}^{3}, \dot{\boldsymbol{r}}_{c} \in \mathbb{R}^{3}$ are the position of the CM of the whole system expressed in $\mathcal{C}$ and its time derivative, respectively; $\phi \in \mathbb{R}^{3}, \dot{\phi} \in \mathbb{R}^{3}$ are the Euler angle vector and its time derivative, respectively; $\boldsymbol{J}_{\phi} \in \mathbb{R}^{3 \times 3}$ is the Jacobian mapping $\boldsymbol{\omega}_{b}$ in $\dot{\phi}$. The matrix $\boldsymbol{\Xi}$ is function of the generalized coordinates $\boldsymbol{y}$. Note that any rotation parameterization different from the Euler angles can be used without affecting the following joint dynamics derivation.

Introducing $\boldsymbol{x}=\left[\begin{array}{lll}\boldsymbol{v}_{c}^{T} & \boldsymbol{h}_{c}^{r T} & \dot{\boldsymbol{q}}^{T}\end{array}\right]^{T} \in \mathbb{R}^{6+n}$ and assuming that 
$\boldsymbol{\Xi}^{T}$ is invertible, the following relation can be written:

$$
\dot{\boldsymbol{y}}=\boldsymbol{\Omega} \boldsymbol{x}
$$

where $\boldsymbol{\Omega}=\boldsymbol{\Xi}^{-T} \in \mathbb{R}^{(6+n) \times(6+n)}$.

Exploiting Eqs. (31)-(32), the quasi-Lagrangian formulation can be derived from the standard one [12], resulting in

$$
\delta \boldsymbol{y}^{T}\left(\boldsymbol{\Xi} \frac{d}{d t}\left[\frac{\partial T}{\partial \boldsymbol{x}}\right]+\dot{\boldsymbol{\Xi}} \frac{\partial T}{\partial \boldsymbol{x}}-\frac{\partial T}{\partial \boldsymbol{y}}-\boldsymbol{H} \frac{\partial T}{\partial \boldsymbol{x}}-\boldsymbol{Q}\right)=0,
$$

where

$$
\boldsymbol{H}=\left[\begin{array}{c}
\boldsymbol{x}^{T} \boldsymbol{\Omega}^{T} \frac{\partial \boldsymbol{\Xi}}{\partial y_{1}} \\
\vdots \\
\boldsymbol{x}^{T} \boldsymbol{\Omega}^{T} \frac{\partial \boldsymbol{\Xi}}{\partial y_{6+n}}
\end{array}\right] \in \mathbb{R}^{(6+n) \times(6+n) ;}
$$

$\boldsymbol{Q} \in \mathbb{R}^{6+n}$ is the vector of the generalized forces; $T \in \mathbb{R}$ is the kinetic energy of the system expressed in terms of $\boldsymbol{x}$. The potential energy is assumed to be zero. $T$ can be computed as follows

$$
T=\frac{1}{2}\left(m \boldsymbol{v}_{c}^{T} \boldsymbol{v}_{c}+\dot{\boldsymbol{q}}^{T} \boldsymbol{M}_{m}^{*} \dot{\boldsymbol{q}}+\boldsymbol{h}_{c}^{r T} \boldsymbol{I}_{c}^{-T} \boldsymbol{h}_{c}^{r}\right) .
$$

The matrices $\dot{\Xi}$ and $\boldsymbol{\Omega}^{T} \frac{\partial \Xi}{\partial y_{i}}$, appearing in Eqs. (33)-(34), can be expanded as

$$
\begin{gathered}
\dot{\boldsymbol{\Xi}}=\left[\begin{array}{ccc}
\mathbf{0} & \mathbf{0} & \mathbf{0} \\
\dot{\boldsymbol{J}}_{\phi}^{-T}\left[\boldsymbol{r}_{c}\right]^{\wedge}+\boldsymbol{J}_{\phi}^{-T}\left[\dot{\boldsymbol{r}}_{c}\right]^{\wedge} & \dot{\boldsymbol{J}}_{\phi}^{-T} \boldsymbol{I}_{c}^{T}+\boldsymbol{J}_{\phi}^{-T} \dot{\boldsymbol{I}}_{c}^{T} & \mathbf{0} \\
\mathbf{0} & \overline{\boldsymbol{J}}_{\omega}^{T} \boldsymbol{I}_{c}^{T}+\overline{\boldsymbol{J}}_{\omega}^{T} \dot{\boldsymbol{I}}_{c}^{T} & \mathbf{0}
\end{array}\right] \\
\boldsymbol{\Omega}^{T} \frac{\partial \boldsymbol{\Xi}}{\partial y_{i}}=\left[\begin{array}{ccc}
\mathbf{0} & \mathbf{0} & \mathbf{0} \\
\boldsymbol{I}_{c}^{-T} \boldsymbol{J}_{\phi}^{T} \frac{\partial\left(\boldsymbol{J}_{\phi}^{-T}\left[\boldsymbol{r}_{c}\right]^{\wedge}\right)}{\partial y_{i}} & \boldsymbol{I}_{c}^{-T} \boldsymbol{J}_{\phi}^{T} \frac{\partial\left(\boldsymbol{J}_{\phi}^{-T} \boldsymbol{I}_{c}^{T}\right)}{\partial y_{i}} & \mathbf{0} \\
-\overline{\boldsymbol{J}}_{\omega}^{T} \boldsymbol{J}_{\phi}^{T} \frac{\partial\left(\boldsymbol{J}_{\phi}^{-T}\left[\boldsymbol{r}_{c}\right]^{\wedge}\right)}{\partial y_{i}} & -\overline{\boldsymbol{J}}_{\omega}^{T} \boldsymbol{J}_{\phi}^{T} \frac{\partial\left(\boldsymbol{J}_{\phi}^{-T} \boldsymbol{I}_{c}^{T}\right)}{\partial y_{i}}+ & \mathbf{0} \\
& \frac{\partial\left(\overline{\boldsymbol{J}}_{\omega}^{T} \boldsymbol{I}_{c}^{T}\right)}{\partial y_{i}}
\end{array}\right.
\end{gathered}
$$

Therefore, the matrix $\boldsymbol{H}$ has this form:

$$
\boldsymbol{H}=\left[\begin{array}{c}
\boldsymbol{x}^{T} \boldsymbol{\Omega}^{T} \frac{\partial \boldsymbol{\Xi}}{\partial y_{1}} \\
\vdots \\
\boldsymbol{x}^{T} \boldsymbol{\Omega}^{T} \frac{\partial \boldsymbol{\Xi}}{\partial y_{6+n}}
\end{array}\right]=\left[\begin{array}{ccc}
\boldsymbol{H}_{t} & \boldsymbol{H}_{t r} & \mathbf{0} \\
\boldsymbol{H}_{r t} & \boldsymbol{H}_{r} & \mathbf{0} \\
\boldsymbol{H}_{m t} & \boldsymbol{H}_{m r} & \mathbf{0}
\end{array}\right]
$$

Since the joint dynamics is of interest, only the last $n$ rows of the Lagrangian system can be considered. Hence, only the last $n$ rows of $\boldsymbol{H}$ are expanded. It can be noted that $\boldsymbol{H}_{m t}=\mathbf{0}$ because neither $\boldsymbol{J}_{\phi}$ nor $\boldsymbol{r}_{c}$ depends on $\boldsymbol{q}$, while $\boldsymbol{H}_{m r}$ can be expanded as follows

$$
\begin{aligned}
\boldsymbol{H}_{m r} & =\left[\begin{array}{c}
\boldsymbol{h}_{c}^{r T} \boldsymbol{I}_{c}^{-T} \frac{\partial \boldsymbol{I}_{c}^{T}}{\partial q_{1}}+\dot{\boldsymbol{q}}^{T} \frac{\partial \overline{\boldsymbol{J}}_{\omega}^{T}}{\partial q_{1}} \boldsymbol{I}_{c}^{T} \\
\vdots \\
\boldsymbol{h}_{c}^{r T} \boldsymbol{I}_{c}^{-T} \frac{\partial \boldsymbol{I}_{c}^{T}}{\partial q_{n}}+\dot{\boldsymbol{q}}^{T} \frac{\partial \overline{\boldsymbol{J}}_{\omega}^{T}}{\partial q_{n}} \boldsymbol{I}_{c}^{T}
\end{array}\right]= \\
& =-\boldsymbol{I}_{c / q}^{-T} \boldsymbol{I}_{c}^{T}+\overline{\boldsymbol{J}}_{\omega / q}^{T} \boldsymbol{I}_{c}^{T},
\end{aligned}
$$

where $\boldsymbol{I}_{c / q}^{-T}, \overline{\boldsymbol{J}}_{\omega / q}^{T}$ are reported in Eqs. (18) and (19), respectively.

Considering that

$$
\frac{\partial T}{\partial \boldsymbol{x}}=\left[\begin{array}{c}
m \boldsymbol{v}_{c} \\
\boldsymbol{I}_{c}^{-T} \boldsymbol{h}_{c}^{r} \\
\boldsymbol{M}_{m}^{*} \dot{\boldsymbol{q}}
\end{array}\right],
$$

$$
\frac{\partial T}{\partial \boldsymbol{y}}=\mathbf{0},
$$

after some simplification, the joint dynamic equation turns out to be

$$
\begin{array}{r}
\overline{\boldsymbol{J}}_{\omega}^{T} \boldsymbol{I}_{c}^{T}\left(\boldsymbol{I}_{c}^{-T} \dot{\boldsymbol{h}}_{c}^{r}+\dot{\boldsymbol{I}}_{c}^{-T} \boldsymbol{h}_{c}^{r}\right)+\boldsymbol{M}_{m}^{*} \ddot{\boldsymbol{q}}+\dot{\boldsymbol{M}}_{m}^{*} \dot{\boldsymbol{q}}+ \\
+\dot{\overline{\boldsymbol{J}}}_{\omega}^{T} \boldsymbol{h}_{c}^{r}-\frac{1}{2}\left(\boldsymbol{M}_{m / q}^{*} \dot{\boldsymbol{q}}-\boldsymbol{I}_{c / q}^{-T} \boldsymbol{h}_{c}^{r}\right)-\overline{\boldsymbol{J}}_{\omega / q}^{T} \boldsymbol{h}_{c}^{r}+ \\
+\overline{\boldsymbol{J}}_{\omega}^{T} \dot{\boldsymbol{I}}_{c}^{T} \boldsymbol{I}_{c}^{-T} \boldsymbol{h}_{c}^{r}=\boldsymbol{\tau}_{t o t}-\overline{\boldsymbol{J}}_{v}^{T} \boldsymbol{f}_{b, t o t}
\end{array}
$$

where $\tau_{\text {tot }} \in \mathbb{R}^{n}$ are the total torques acting on the joints, i.e., including commanded torques and disturbance torques; $f_{b, t o t} \in \mathbb{R}^{n}$ are the total forces acting on the base, i.e., including commanded forces and disturbance forces.

Substituting $\dot{\boldsymbol{h}}_{c}^{r}$ from Eq. (30) and $\dot{\boldsymbol{I}}_{c}^{T}=-\boldsymbol{I}_{c}^{T} \dot{\boldsymbol{I}}_{c}^{-T} \boldsymbol{I}_{c}^{T}$, and using relations (13) and (14), the dynamics (42) can be rewritten as

$$
\begin{array}{r}
\boldsymbol{M}_{m}^{*} \ddot{\boldsymbol{q}}+\left(\dot{\boldsymbol{M}}_{m}^{*}-\frac{1}{2} \boldsymbol{M}_{m / q}^{*}\right) \dot{\boldsymbol{q}}+\left(\dot{\overline{\boldsymbol{J}}}_{\omega}^{T}-\overline{\boldsymbol{J}}_{\omega}^{T}\left[\boldsymbol{\omega}_{b}\right]^{\wedge}+\right. \\
\left.+\frac{1}{2} \boldsymbol{I}_{c / q}^{-T}-\overline{\boldsymbol{J}}_{\omega / q}^{T}\right) \boldsymbol{h}_{c}^{r}=\overline{\boldsymbol{\tau}}_{t o t},
\end{array}
$$

where $\bar{\tau}_{t o t}=\bar{\tau}+\bar{\tau}_{\text {ext }} \in \mathbb{R}^{n}$.

Finally, comparing Eq. (43) and the third row of Eq. (15), the relation (16c) can be obtained.

\section{REFERENCES}

[1] B. Siciliano and O. Khatib, Springer handbook of robotics. Springer, 2016.

[2] S. Abiko, R. Lampariello, and G. Hirzinger, "Impedance control for a free-floating robot in the grasping of a tumbling target with parameter uncertainty," in Intelligent Robots and Systems, 2006 IEEE/RSJ International Conference on. IEEE, 2006, pp. 1020-1025.

[3] D. N. Nenchev and K. Yoshida, "Impact analysis and post-impact motion control issues of a free-floating space robot subject to a force impulse," IEEE Transactions on Robotics and Automation, vol. 15, no. 3, pp. 548-557, 1999.

[4] A. M. Giordano, G. Garofalo, and A. Albu-Schaffer, "Momentum dumping for space robots," in Decision and Control (CDC), 2017 IEEE 56th Annual Conference on. IEEE, 2017, pp. 5243-5248.

[5] A. Flores-Abad, M. Nandayapa, and M. A. Garcia-Teran, "Force sensorless impedance control for a space robot to capture a satellite for on-orbit servicing," in 2018 IEEE Aerospace Conference. IEEE, 2018.

[6] S. A. A. Moosavian and E. Papadopoulos, "On the control of space free-flyers using multiple impedance control," in Robotics and $A u-$ tomation, 1997. Proceedings., 1997 IEEE International Conference on, vol. 1. IEEE, 1997, pp. 853-858.

[7] F. Flacco, A. Paolillo, and A. Kheddar, "Residual-based contacts estimation for humanoid robots," in Humanoid Robots (Humanoids), 2016 IEEE-RAS 16th International Conference on. IEEE, 2016, pp. $409-415$.

[8] S. Haddadin, A. De Luca, and A. Albu-Schäffer, "Robot collisions: A survey on detection, isolation, and identification," IEEE Transactions on Robotics, vol. 33, no. 6, pp. 1292-1312, 2017.

[9] R. M. Murray, A mathematical introduction to robotic manipulation. CRC press, 2017.

[10] A. M. Giordano, D. Calzolari, and A. Albu-Schäffer, "Workspace fixation for free-floating space robot operations," in Robotics and Automation (ICRA), 2018 IEEE International Conference on. IEEE, 2018.

[11] J. L. Crassidis and F. L. Markley, "Unscented filtering for spacecraft attitude estimation," Journal of guidance, control, and dynamics, vol. 26, no. 4, pp. 536-542, 2003.

[12] J. M. Cameron and W. J. Book, "Modeling mechanisms with nonholonomic joints using the Boltzmann-Hamel equations," The International Journal of Robotics Research, vol. 16, no. 1, pp. 47-59, 1997. 